\title{
Non-invasive Vagus Nerve Stimulation (nVNS) as mini-prophylaxis for menstrual/ menstrually related migraine: an open-label study
}

Licia Grazzi ${ }^{1}$, Gabriella Egeo ${ }^{2}$, Anne H. Calhoun ${ }^{3}$, Candace K. McClure ${ }^{4}$, Eric Liebler ${ }^{5}$ and Piero Barbanti ${ }^{*}$

\begin{abstract}
Background: Menstrual migraine and menstrually related migraine attacks are typically longer, more disabling, and less responsive to medications than non-menstrual attacks. The aim of this study was to evaluate the efficacy, safety, and tolerability of non-invasive vagus nerve stimulation for the prophylactic treatment of menstrual migraine/menstrually related migraine.

Methods: Fifty-six enrolled subjects (menstrual migraine, 9 \%; menstrually related migraine, $91 \%$ ), 33 (59 \%) of whom were receiving other prophylactic therapies, entered a 12-week baseline period. Fifty-one subjects subsequently entered a 12-week treatment period to receive open-label prophylactic non-invasive vagus nerve stimulation adjunctively $(31 / 51 ; 61 \%)$ or as monotherapy $(20 / 51 ; 39 \%)$ on day -3 before estimated onset of menses through day +3 after the end of menses.

Results: The number of menstrual migraine/menstrually related migraine days per month was significantly reduced from baseline (mean \pm standard error, $7.2 \pm 0.7$ days) to the end of treatment (mean \pm standard error, 4.7 \pm 0.5 days; $P<0.001$ ) (primary end point). Of all subjects, $39 \%$ (95\% confidence interval: $26 \%, 54 \%)(20 / 51)$ had $a \geq 50 \%$ reduction (secondary end point). For the other secondary end points, clinically meaningful reductions in analgesic use (mean change \pm standard error, $-3.3 \pm 0.6$ times per month; $P<0.001$ ), 6 -item Headache Impact Test score (mean change \pm standard error, $-3.1 \pm 0.7 ; P<0.001$ ), and Migraine Disability Assessment score (mean change \pm standard error, $-11.9 \pm 3.4 ; P<0.001$ ) were observed, along with a modest reduction in pain intensity (mean change \pm standard error, $-0.5 \pm 0.2 ; P=0.002$ ). There were no safety/tolerability concerns.

Conclusions: These findings suggest that non-invasive vagus nerve stimulation is an effective treatment that reduces the number of menstrual migraine/menstrually related migraine days and analgesic use without safety/tolerability concerns in subjects with menstrual migraine/menstrually related migraine. Randomised controlled studies are warranted.
\end{abstract}

Keywords: Menstrual migraine, Menstrually related migraine, Prophylactic treatment, Vagus nerve

\footnotetext{
* Correspondence: piero.barbanti@sanraffaele.it

${ }^{2}$ Headache and Pain Unit, Istituto di Ricovero e Cura a Carattere Scientifico,

San Raffaele Pisana, Via della Pisana 235, 00163 Rome, Italy

Full list of author information is available at the end of the article
} 


\section{Background}

Menstrual migraine (MM) without aura is defined as the exclusive occurrence of attacks on days -2 to +3 of menstruation in at least 2 of 3 consecutive menstrual cycles according to the International Classification of Headache Disorders, 3rd edition (beta version) (ICHD-III beta) appendix (i.e., requires further validation), and menstrually related migraine (MRM) without aura is also characterized by the occurrence of attacks on other days of the cycle [1]. More than $90 \%$ of women with migraine attacks during menstruation have MRM [2]; the estimated prevalence among migraineurs has varied from 0.85 to $14.1 \%$ for $\mathrm{MM}$ and from 3 to $71.4 \%$ for MRM [3]. These conditions are believed to be a result of fluctuating oestrogen levels; steady or elevating levels are associated with a protective effect, whereas abrupt oestrogen withdrawals are associated with precipitation of migraine attacks $[2,4]$. In the late luteal phase of the menstrual cycle, decreased oestrogen levels have been observed, which lead to serotonin declines and are likely responsible for the triggering of $\mathrm{MM} / \mathrm{MRM}$ attacks just prior to menses [2, 4].

No acute or prophylactic therapies are currently approved specifically for the treatment of MM/MRM in the European Union or the United States [5, 6]. Acute treatments used for these conditions are the same as those used for non-menstrual migraine and include triptans, non-steroidal anti-inflammatory drugs (NSAIDs), and ergot derivatives $[2,6]$. Prophylaxis comprises shortterm and continuous treatments [2]. Short-term prophylactic therapies are administered only during the time of need (e.g., perimenstrually) and include triptans, oestrogen, and naproxen, whereas continuous prophylactic options such as hormonal contraceptives provide ongoing exposure to the treatment [2]. A systematic review of $M M / M R M$ clinical trials indicated that evidence supporting most categories of prophylactic MM/MRM treatments is generally weak [6]. Clinical studies of triptans represent the strongest evidence to date for acute and preventive MM/MRM treatment, which supports almotriptan, naratriptan, sumatriptan, and zolmitriptan as acute therapies and frovatriptan, naratriptan, and zolmitriptan as preventive therapies [6].

Despite the general safety and tolerability of triptans with appropriate patient selection $[2,7,8]$, the MM/ MRM population may have unique challenges related to the adverse events (AEs) associated with these treatments. Compared with non-menstrual migraine attacks, MM/MRM attacks are generally longer lasting, more debilitating, more prone to recurrence, and less responsive to therapies such as triptans $[9,10]$. Results from a large study showed that $44 \%$ and $48 \%$ of migraineurs were dissatisfied with triptan-associated tolerability and general/work-related functional ability, respectively [7], and these concerns may be even more prominent in the relatively treatment-refractory MM/MRM population [10]. Menses is generally considered to be a period of inherent discomfort [11], and the repeated treatments required to mitigate the effects of $M M / M R M$ could further exacerbate the level of discomfort while providing only minimal response $[10,12]$. Evidence-based guidelines for migraine suggest limiting the use of triptans to 2 headache days per week to reduce the risk of rebound or medication-overuse headache [8], and frequent use of these agents may lead to misuse/overuse and has been significantly associated with the development of chronic migraine $[13,14]$. Thus, the prophylactic administration of triptans over the course of several days during menstruation coupled with the potential need for acute triptan therapy in women with MM/MRM could complicate the condition $[12,13]$. Patients may be unwilling to accept the AE burden and/or potential complications of adhering to monthly prophylactic MM/MRM therapies [15], defining a need for alternative treatment options among this population.

Non-invasive vagus nerve stimulation (nVNS) (gammaCore ${ }^{\circledR}$; electroCore, LLC; Basking Ridge, NJ, USA) provides neuromodulation that transfers electrical impulses transcutaneously to the cervical branch of the vagus nerve. In 4 open-label $\leq 12$-week studies, nVNS demonstrated efficacy, safety, and tolerability as an acute/ prophylactic therapy for migraine and chronic cluster headache [16-19]. Based on the treatment benefits observed in previous studies and the potential for reducing medication overuse and medication-associated AEs [16], we evaluated nVNS used as mini-prophylaxis for $M M / M R M$ in this 24-week study of 56 subjects.

\section{Methods \\ Study design}

This investigator-initiated, multicentre, single-arm, open-label study of the efficacy, safety, and tolerability of prophylactic nVNS treatment for MM/MRM was conducted from January 2015 to October 2015. After providing informed consent for trial participation and publication of patient data, subjects entered a 12-week run-in (baseline) period of observation followed by a 12 -week nVNS treatment period to receive open-label, short-term MM/MRM prophylaxis. Investigators obtained approval from the Ethical Committees of the Istituto di Ricovero e Cura a Carattere Scientifico (IRCCS), San Raffaele Pisana (identifier: SR_MM12/ 2014). All source documents and files are stored in the clinical trial centres.

\section{Subjects}

All subjects were enrolled from two Italian sites and were required to have a regular menstrual cycle. Inclusion 
criteria specified that subjects were to be 18 to 50 years of age. Subjects had a $>1$-year history of migraine with or without aura and a diagnosis of MM/MRM without aura according to ICHD-III beta criteria [1]. Key exclusion criteria at the time of enrolment were another diagnosis of a primary headache disorder, such as chronic migraine, secondary headache disorder, or other neurological or severe systemic disease; current or previous vagal disturbances; a change in prophylactic medication type or dosage within 1 month before enrolment; previous failure of $\geq 3$ prophylactic treatment classes; and participation in another clinical trial.

\section{Intervention}

The nVNS devices (Fig. 1) for this study, supplied by electroCore, LLC, produce a proprietary low-voltage electrical signal comprising a $5-\mathrm{kHz}$ sine wave burst lasting for 1 millisecond (five sine waves, each lasting 200 microseconds), with such bursts repeated once every 40 milliseconds $(25 \mathrm{~Hz})$, generating a $24-\mathrm{V}$ peak voltage and 60-mA peak output current. Subjects received comprehensive training on appropriate device placement and adjustment of stimulation intensity for optimal dose administration. Bilateral 2-min stimulations were administered to the cervical branch of the vagus nerve 3 times daily at $8 \mathrm{AM}, 1 \mathrm{PM}$, and $8 \mathrm{PM}$ (i.e., 6 stimulations total per day) after application of conductive gel to the 2 stainless steel contact surfaces. This procedure was performed from -3 days before estimated onset of menstruation through +3 days after the end of menstruation during each cycle of the 12-week nVNS treatment period. Treatment duration per month in this study was 10 to 14 days based on the actual days of menstruation. Doses of any prophylactic medications were kept stable throughout the study. Subjects were allowed to use their usual acute analgesics (alone or in combination), which

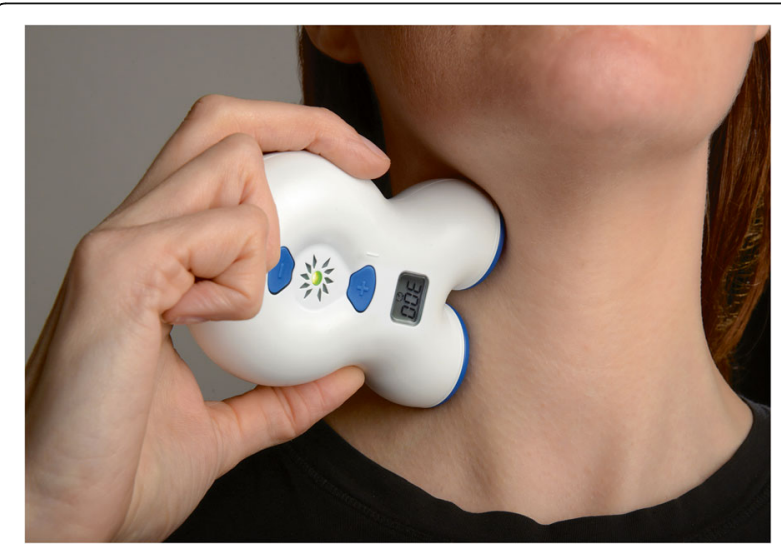

Fig. 1 Non-invasive Vagus Nerve Stimulation Device. Image provided courtesy of electroCore, LLC included triptans, NSAIDs, and a butalbital/caffeine/propyphenazone combination medication.

\section{Study end points}

The primary end point was the mean change in number of MM/MRM days per month between the baseline and nVNS treatment periods. Secondary end points included the proportion of subjects with $a \geq 50 \%$ reduction in the mean number of MM/MRM days from the baseline period to the nVNS treatment period and mean changes between the two study periods in analgesic use, migraine disability, pain intensity, and allodynia. Exploratory analyses of proportions of subjects who achieved various levels of improvement in migraine disability were also performed.

\section{Data collection}

At baseline, subjects underwent a physical and neurological examination and a live interview administered via a detailed, semistructured questionnaire on their 1) clinical migraine features; 2) demographics, lifestyle, and behavioural factors; and 3) comorbidities and concomitant medications [20]. During the study periods, subjects used diary cards to record their MM/MRM days and analgesic use on a daily basis. This information was used to calculate monthly averages for each 3-month study period. Data on migraine disability were collected using the 6-item Headache Impact Test $\left(\right.$ HIT- $\left.6^{\mathrm{TM}}\right)$ [21] based on the last 4 weeks of each study period and the MIDAS based on the entire 3 months of each study period [22]. Scores on the HIT- 6 range from 36 to 78 points, MIDAS scores range from 0 to $21+$ points, and MIDAS grades range from 1 to 4 , with higher values indicating greater disability for each of these assessments [21, 22]. Pain intensity for each migraine attack was rated using question $\mathrm{B}$ of the Migraine Disability Assessment (MIDAS) on a scale from 0 to 10 , with higher values indicating greater intensity, and was averaged at the end of each 3-month study period [22]. Data on allodynia were collected using the 12-item Allodynia Symptom Checklist (ASC-12), with scores ranging from 0 to 24 points and higher scores indicating greater severity [23]. The investigator collected safety and tolerability data by asking participants to provide subjective responses at each study visit regarding any AEs after nVNS use.

\section{Statistical analyses}

Subjects who received at least $1 \mathrm{nVNS}$ treatment were included in the intent-to-treat (ITT) population for efficacy analyses. Mean \pm standard error of the mean (SEM) changes between the two study periods were calculated using data from week 12 of the nVNS treatment period minus data from the baseline visit, with negative values indicating a decrease over time. Two-sided statistical 
analyses were conducted using a significance level of $P<$ $0.05 ; P$ values were derived from the $t$ test. The exact Clopper-Pearson method was used to calculate the $95 \%$ confidence interval (CI) for the proportion of subjects with $a \geq 50 \%$ reduction in mean number of $M M / M R M$ days between the two study periods. Missing data were imputed using the last observation carried forward method. Data for subjects who discontinued during the nVNS treatment period were imputed to baseline values and were included in all efficacy analyses, whereas subjects with missing data for both study periods were not included in the analyses. All statistical analyses were performed independently by North American Science Associates Inc. (Minneapolis, MN, USA) using SAS ${ }^{\oplus} 9.3$ (SAS Institute Inc., Cary, NC, USA).

\section{Results}

Subjects

Of the 56 subjects screened, all were enrolled and entered the baseline period, including 6 subjects who were older than 50 years of age but met all other entry criteria. All subjects were diagnosed with MM/MRM without aura and had episodic migraine at enrolment. Demographic and baseline characteristics (Table 1) showed a mean age of 40.2 years. Most subjects had MRM (91\%), and the remaining $9 \%$ had MM. For 7 subjects, the average number of headache days per month in the baseline period had increased to a range of 15 to 20 days; this increase was not sustained for more than 3 months, thereby excluding a diagnosis of chronic migraine. Five subjects discontinued from the study before entering the nVNS treatment period, including 2 subjects who became pregnant, 1 subject who entered menopause, 1 subject who underwent surgery, and 1 subject for whom no reason for study withdrawal was provided. The remaining 51 subjects met criteria for the ITT population. Of these, 5 subjects discontinued from the nVNS treatment period because of lack of efficacy, and 1 subject discontinued because of non-device-related AEs.

\section{Menstrual/Menstrually related migraine days}

Therapy with nVNS significantly reduced the mean number of MM/MRM days (primary end point) from baseline (7.2 \pm 0.7 days) to the end of the treatment period (4.7 \pm 0.5 days; $P<0.001$ ) (Fig. 2). During the nVNS treatment period, $39 \%$ (95\% CI: $26 \%$, 54\%) of all subjects $(20 / 51)$ had $a \geq 50 \%$ reduction in the mean number of MM/MRM days from baseline.

\section{Analgesic use}

Mean analgesic use was significantly reduced from $8.9 \pm 0.8$ times per month in the baseline period to
Table 1 Demographic and Baseline Characteristics ${ }^{a}$

\begin{tabular}{|c|c|}
\hline Characteristic & $\begin{array}{l}\text { Total Population } \\
(n=56)\end{array}$ \\
\hline Age $(y)$, mean \pm SEM & $40.2 \pm 1.0$ \\
\hline Age of onset $(y)$, mean \pm SEM & $17.9 \pm 1.3$ \\
\hline Currently employed, No. (\%) & $49(88)$ \\
\hline \multicolumn{2}{|l|}{ Migraine type, No. (\%) } \\
\hline MRM & $51(91)$ \\
\hline MM & $5(9)$ \\
\hline \multicolumn{2}{|l|}{ Pain location, No. (\%) } \\
\hline Bilateral & $17(30)$ \\
\hline Unilateral & $39(70)$ \\
\hline \multicolumn{2}{|l|}{$\begin{array}{l}\text { Average duration of untreated/unsuccessfully } \\
\text { treated attacks, No. (\%) }\end{array}$} \\
\hline$<12 \mathrm{~h}$ & $2(4)$ \\
\hline 12 to $<24 h$ & $15(27)$ \\
\hline 24 to $<48 h$ & $7(13)$ \\
\hline 48 to $<72 h$ & $14(25)$ \\
\hline$\geq 72 \mathrm{~h}$ & $18(32)$ \\
\hline \multicolumn{2}{|l|}{ Analgesic medications used, No. (\%) } \\
\hline NSAID & $17(30)$ \\
\hline NSAID plus triptan & $20(36)$ \\
\hline $\begin{array}{l}\text { NSAID plus a butalbital/caffeine/propyphenazone } \\
\text { combination medication }\end{array}$ & $1(2)$ \\
\hline Triptan & $18(32)$ \\
\hline Other prophylactic medication use, No. $(\%)^{b}$ & $33(59)$ \\
\hline
\end{tabular}

Abbreviations: $M M$, menstrual migraine, MRM menstrually related migraine, NSAID nonsteroidal anti-inflammatory drug, nVNS non-invasive vagus nerve stimulation, SEM standard error of the mean

${ }^{\mathrm{a}}$ Data represent values from the baseline period

${ }^{b}$ Amitriptyline (5/56), coenzyme Q10/ginkgolide B/riboflavin (tablets) plus magnesium (4/56), coenzyme Q10/ginkgolide B/riboflavin/magnesium (granulated) (1/56), coenzyme Q10/ginkgolide B/riboflavin/magnesium (granulated) plus amitriptyline (1/56), magnesium (3/56), $\beta$-blocker (2/56), calcium channel blocker (2/56), paroxetine (2/56), riboflavin (2/56), amitriptyline plus benzodiazepine (1/56), amitriptyline plus magnesium (1/56), $\beta$-blocker plus amitriptyline (1/56), botulinum toxin $A(1 / 56)$, sodium valproate plus venlafaxine $(1 / 56)$, sodium valproate plus paroxetine $(1 / 56)$, tanacetum parthenium $(1 / 56)$, sertraline $(1 / 56)$, topiramate plus sertraline $(1 / 56)$, topiramate plus gabapentin $(1 / 56)$

$5.6 \pm 0.5$ times per month in the nVNS treatment period $(P<0.001)$ (Fig. 3$)$.

\section{Migraine disability}

A reduction in migraine disability was demonstrated by the mean HIT-6 score being significantly improved from baseline $(67.2 \pm 0.7)$ to the end of the nVNS treatment period (64.1 $\pm 0.7 ; P<0.001)$ (Fig. 4). This improvement of 3.1 exceeds the estimated minimally important difference (MID) of 2.3 to 2.7 that has been established as clinically meaningful [21].

Significant improvements after nVNS treatment were also observed for MIDAS score (baseline, 42.2 \pm 4.7 ; nVNS, $30.3 \pm 3.3 ; P<0.001$ ) (Fig. 5a) and MIDAS grade (baseline, $3.6 \pm 0.1$; nVNS, $3.3 \pm 0.1 ; P=0.008$ ) (Fig. 5b). 


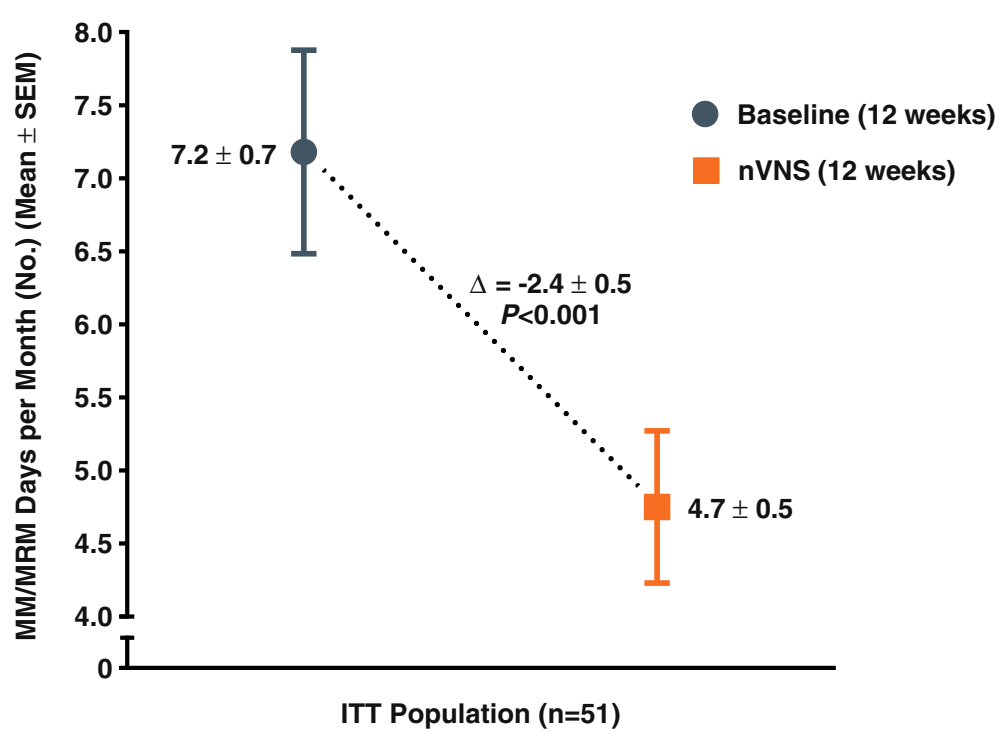

Fig. 2 Change in Number of MM/MRM Days per Month (ITT Population) ${ }^{\text {a. }}$ Abbreviations: ITT, intention-to-treat; nVNS, non-invasive vagus nerve stimulation; SEM, standard error of the mean. ${ }^{a}$ Data are mean $\pm \mathrm{SEM}$; mean differences between treatment groups may not reflect calculated differences because of rounding; $P$ values are from the $t$ test; missing data were imputed using the last observation carried forward

\section{Pain intensity}

Mean pain intensity demonstrated a clinically modest reduction from baseline $(7.6 \pm 0.2)$ to the end of the nVNS treatment period $(7.1 \pm 0.2 ; P=0.002)$ (Fig. 6).

\section{Allodynia}

Allodynia scores during the treated migraine attacks were similar between the two study periods, with a mean difference of $0.2 \pm 0.4$ between the baseline period $(5.4 \pm 0.7)$ and the nVNS treatment period $(5.5 \pm 0.8 ; P=0.58)$.

\section{Safety and tolerability}

There were no safety concerns during the study. The most commonly self-reported AEs were mild or moderate application site reactions (e.g., tingling) and facial/ neck twitching. These AEs were transient in nature, coincided with the stimulation period, and resolved during treatment. No serious AEs occurred. Only 1 subject discontinued from the nVNS treatment period because of non-device-related AEs, which included dizziness and anxiety.

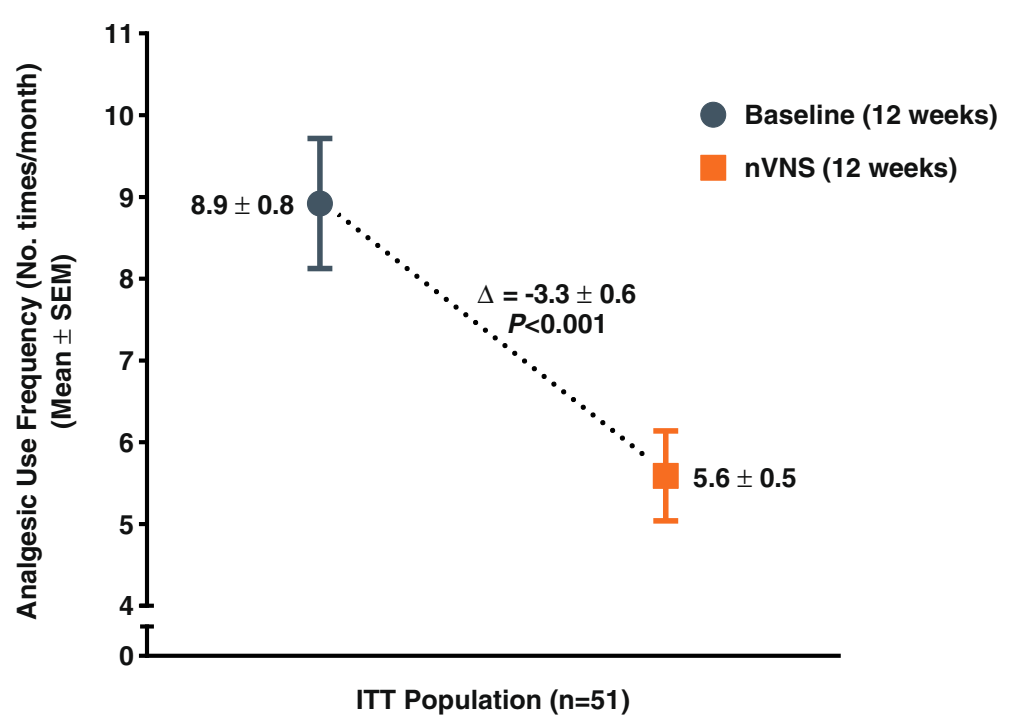

Fig. 3 Change in Analgesic Use per Month (ITT Population) ${ }^{\text {a. }}$ Abbreviations: ITT, intention-to-treat; nVNS, non-invasive vagus nerve stimulation; SEM, standard error of the mean. ${ }^{a}$ Data are mean \pm SEM; mean differences between treatment groups may not reflect calculated differences because of rounding; $P$ values are from the $t$ test; missing data were imputed using the last observation carried forward 


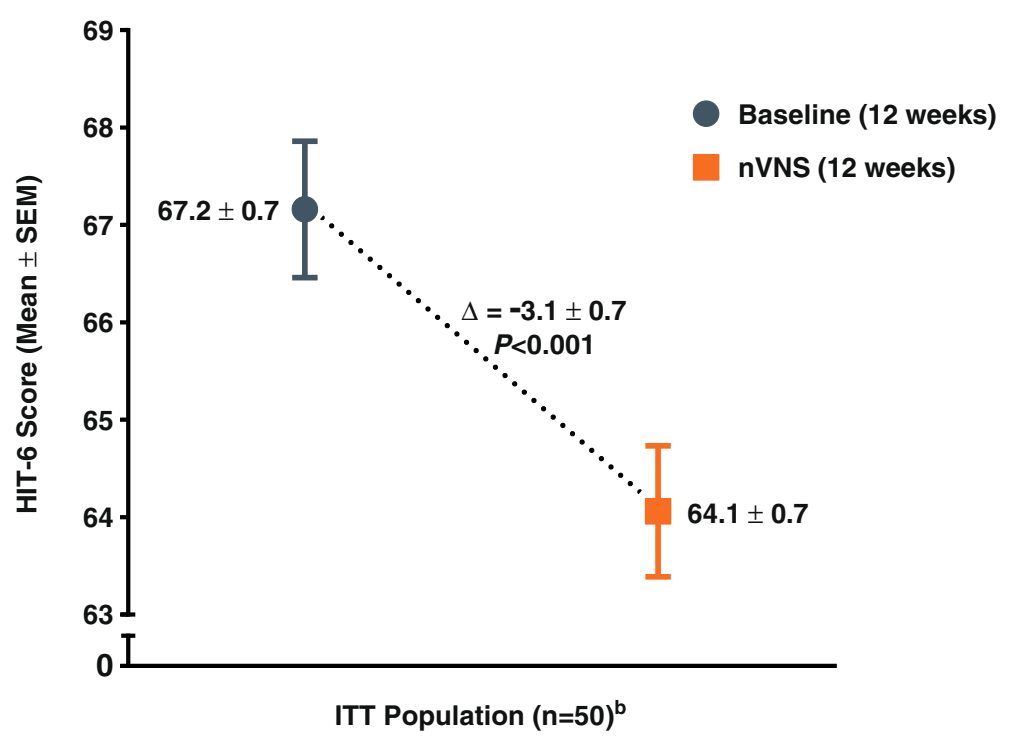

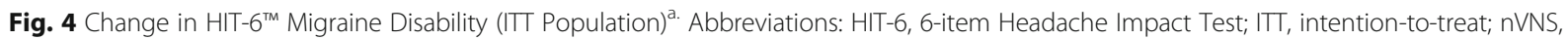
non-invasive vagus nerve stimulation; SEM, standard error of the mean. ${ }^{a}$ Data are mean \pm SEM; mean differences between treatment groups may not reflect calculated differences because of rounding; $P$ values are from the $t$ test; missing data were imputed using the last observation carried forward. ${ }^{b}$ One subject had missing HIT-6 data for both the baseline and nVNS periods

\section{Discussion}

To our knowledge, this study is the first to evaluate the effects of a neuromodulation device for the treatment of MM/MRM without aura. Primary end point results demonstrated that prophylactic nVNS administered from -3 days before estimated onset of menstruation through +3 days after the end of menstruation (a total of 10 to 14 days of treatment per month) for 3 cycles provided an average decrease of 2.4 MM/MRM days per month ( $33 \%)$, which exceeds the MID of 1 headache day established for this end point $[24,25]$. The change in number of headache days from baseline is among the few headache-symptom measures with an established MID, and this MID has been associated with significant effects on health-related quality of life [24, 25]. The assessment of MM/MRM days for the primary end point is clinically relevant to patients and treating physicians, acknowledging that we did not concurrently assess the frequency of individual attacks in this initial study of MM/MRM prophylaxis. The reduction in MM/MRM days was accompanied by significant and clinically meaningful reductions in analgesic use and functional disability. Although this prophylactic study was not intended to evaluate the efficacy of nVNS in the acute treatment of attacks, a modest decrease in pain intensity was observed for the attacks that were not prevented. As several end points in this study represent monthly averages during each 3-month study period, the possibility for improvements in actual monthly MM/MRM outcomes with nVNS treatment over time warrants further evaluation.
Study limitations include the open-label, single-arm study design and the potential for inaccurate subject predictions of menses onset. The open-label study design likely contributed a placebo response to the treatment, as seen in other therapeutic migraine studies $[18,26]$. In this study, the actual treatment effect of nVNS cannot be separated from the potential placebo effect because of the lack of a sham control group. On the basis of the ease of use and lack of AE concerns associated with nVNS in the current study, the device appears to have a favourable risk-benefit profile in $\mathrm{MM} / \mathrm{MRM}$ that is appropriate for further evaluation. As in any MM/MRM study of prophylaxis, imprecise treatment initiation due to inaccurate predictions of the start of menses may have affected the observed results [12] but is likely reflective of real-world use; nVNS has the flexibility to be used outside the perimenstrual period (e.g., before or after menstruation) without increasing the potential for medicationrelated AEs and complications.

The relative refractoriness of MM/MRM attacks to pharmacologic treatments currently available (but not specifically approved) for the condition [10] may be related to the complexity of the relationship between migraine and oestrogen and the brain's sensitivity to fluctuations in hormones that cross the blood-brain barrier [27]. Chronic exposure to oestrogen has been suggested to enhance susceptibility to migraine by promoting increased numbers and excitation of glutamatergic neurons [28]. Paradoxically, oestrogen withdrawal is associated with the occurrence of MM/MRM attacks [27]. 

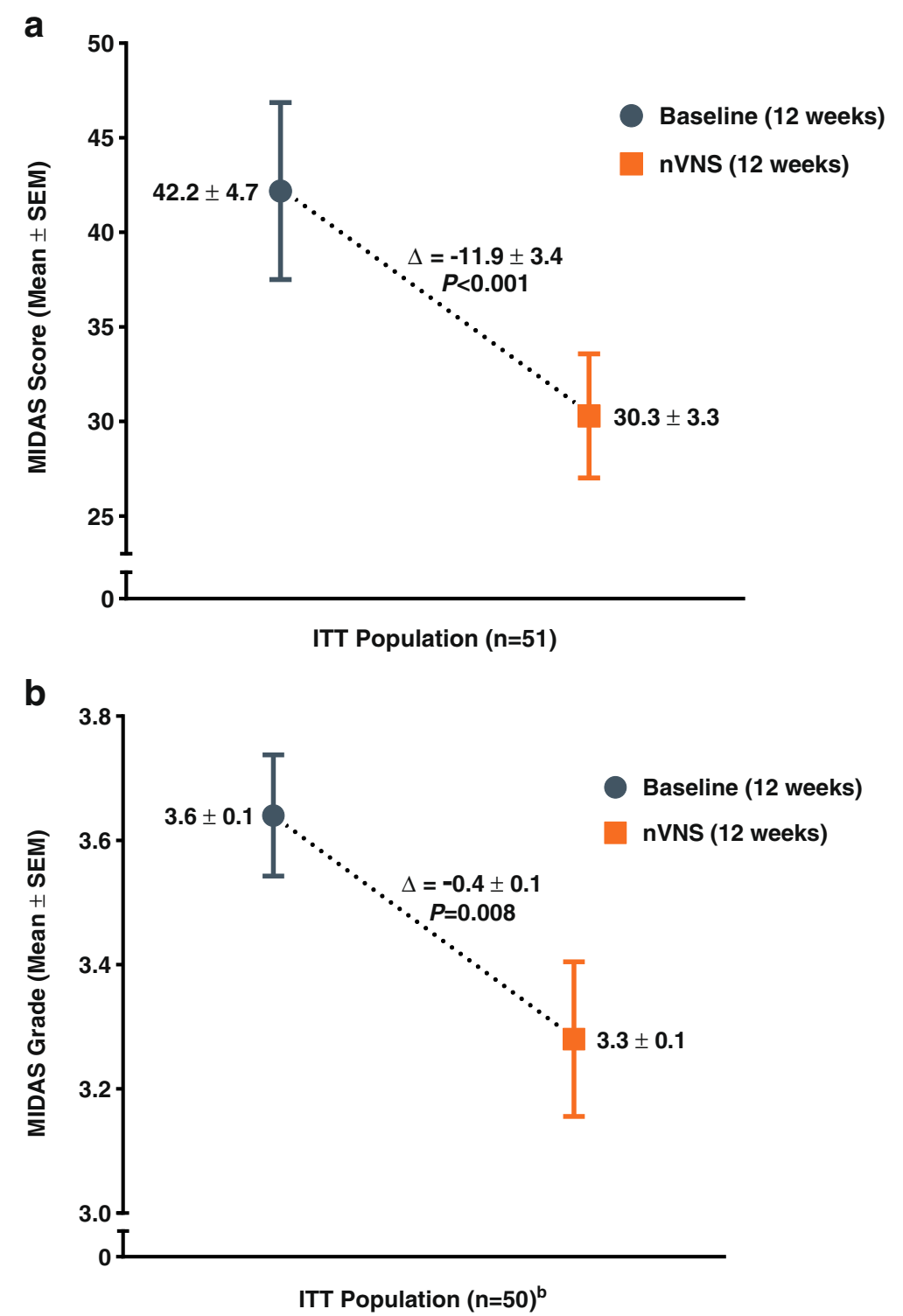

Fig. 5 Change in MIDAS Migraine Disability (ITT Population) ${ }^{\mathrm{a}}$. Abbreviations: ITT, intention-to-treat; MIDAS, Migraine Disability Assessment; $n V N S$, non-invasive vagus nerve stimulation; SEM, standard error of the mean. MIDAS score (a) and MIDAS grade (b). ${ }^{a}$ Data are mean \pm SEM; mean differences between treatment groups may not reflect calculated differences because of rounding; $P$ values were from the $t$ test; missing data were imputed using the last observation carried forward. ${ }^{b}$ One subject had missing MIDAS grade data for both the baseline and nVNS periods

The efficacy of nVNS in the treatment of migraine may be related to the suppression of excessive extracellular levels of glutamate [29]. In MM/MRM, prophylactic nVNS may exert its anti-glutamatergic mechanistic actions directly via the trigeminal nociceptive pathways of women who are susceptible to oestrogen-related attacks.

The efficacy of prophylactic medications used for MM/MRM has been demonstrated in clinical trials $[30,31]$ but may be limited by oestrogen level declines and by concerns related to rebound headaches, practicality, and safety. Managing optimal oestrogen levels is challenging for many clinicians, especially in the context of orally administered drugs, because of the complex changes that occur throughout the menstrual cycle [27]. Randomised, double-blind, placebo-controlled MM/MRM studies have suggested that prophylactic trip$\tan$ and perimenstrual oestrogen therapy may lead to post-treatment or rebound migraine attacks [32-34], possibly reflecting a delayed abrupt decrease in oestrogen levels upon medication discontinuation or a nonhormonal occurrence of end-menstrual migraine (i.e., related to blood loss and transient relative anaemia) [35]. Rebound attacks were not observed after nVNS discontinuation in the current trial or in previous studies of 


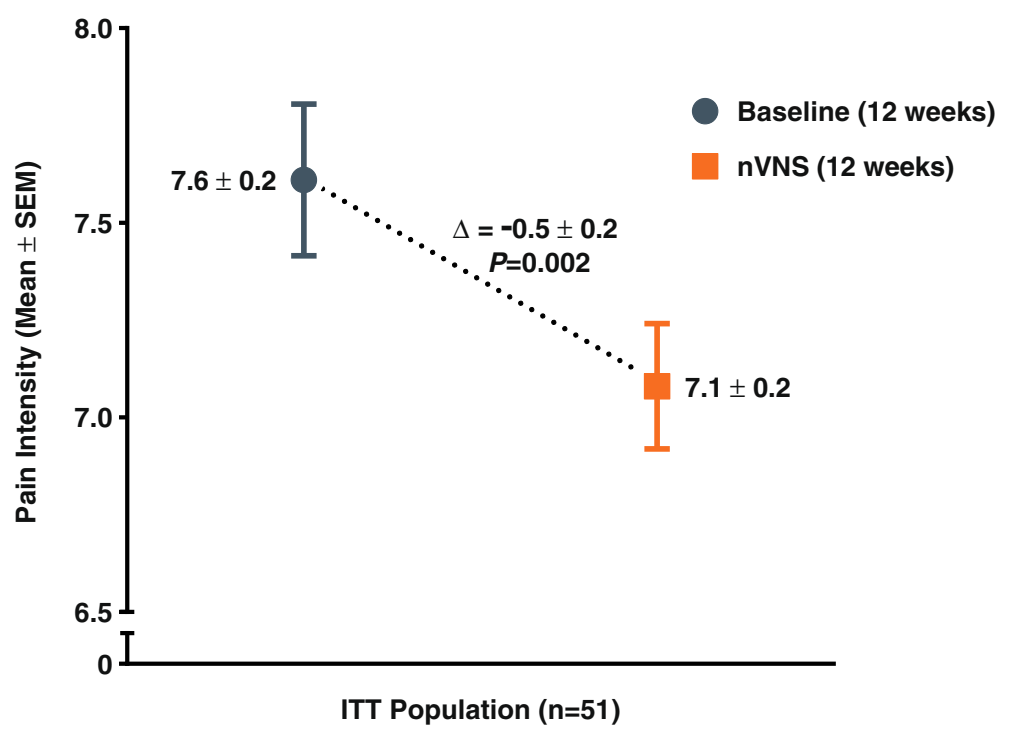

Fig. 6 Change in Pain Intensity (ITT Population) a. Abbreviations: ITT, intention-to-treat; nVNS, non-invasive vagus nerve stimulation; SEM, standard error of the mean. ${ }^{a}$ Data are mean \pm SEM; mean differences between treatment groups may not reflect calculated differences because of rounding; $P$ values are from the $t$ test; missing data were imputed using the last observation carried forward

primary headache conditions [16-19], but additional studies are needed to rule out the potential for post-nVNS migraines. Patients with MM/MRM may be reluctant to accept these and other AE-related consequences of preventive triptans given the potentially modest therapeutic gain $[12,15,32]$. The oral anticonvulsant topiramate has been shown to be effective in the prevention of MM attacks but does not appear to reduce attack severity or duration [36]. In addition, topiramate may require several months of daily administration to achieve its potential benefits and may increase the risk for serious adverse events [36, 37]. Non-invasive vagus nerve stimulation is a practical nonpharmacologic option that does not affect oestrogen levels, interact with acute medications, or increase cardiovascular disease risk. Results of the current study suggest that prophylactic nVNS reduces medication use in women with MM/MRM, thereby possibly mitigating the future risk of medication overuse headache and chronic migraine $[13,16,38]$.

Non-invasive vagus nerve stimulation may serve as an effective therapeutic option with no safety or tolerability concerns for MM/MRM prevention and may mitigate the risk of medication-related AEs and complications, rebound headaches, and potential drug interactions that are highly relevant among this population. Prophylactic use of nVNS for up to 14 days per month is practical and appears to provide a clinical benefit in MM/MRM while decreasing the need for analgesic medications, consistent with results of previous nVNS studies of other primary headache disorders [16-19].

\section{Conclusions}

This study suggests that mini-prophylaxis with nVNS is an effective treatment that reduces the number of MM/ MRM days and acute analgesic use for subjects with MM/MRM without adding any treatment-related safety or tolerability concerns. The current study also expands the body of evidence regarding this condition, for which there are no specifically approved therapies. As the first neuromodulation technique evaluated in patients with MM/MRM, nVNS may offer an important new option for effectively treating the condition while mitigating the monthly perimenstrual AE burden and potential complications of pharmacologic medications. Randomised controlled studies are needed to validate these results.

\section{Abbreviations}

AE: Adverse event; ASC-12: Allodynia symptom checklist; Cl: Confidence interval; HIT-6 ${ }^{\text {TM: }}$ 6-item Headache Impact Test; ICHD-III beta: International classification of headache disorders, 3rd edition (beta version); IRCCS: Istituto di Ricovero e Cura a Carattere Scientifico; ITT: Intent-to-treat; MID: Minimally important difference; MIDAS: Migraine disability assessment; MM: Menstrual migraine; MRM: Menstrually related migraine; NSAID: Non-steroidal anti-inflammatory drug; nVNS: Non-invasive vagus nerve stimulation; SEM: Standard error of the mean

\section{Acknowledgements}

The authors would like to acknowledge Anna Maria Padovan for her role in patient training for the study. Professional writing and editorial support was provided by Stefanie Dorlas, BMath, BEd, of MedLogix Communications, LLC, under the direction of the authors.

\section{Funding}

This study was investigator initiated. Study devices were supplied free of charge by electroCore, LLC (Basking Ridge, NJ, USA). Statistical analyses for the study were conducted by Candace McClure, PhD, of North American Science Associates Inc. (Minneapolis, MN, USA) and were supported by 
electroCore, LLC. Professional writing and editorial support from MedLogix Communications, LLC (Schaumburg, IL, USA), funded by electroCore, LLC, was provided under the direction of the authors throughout draft development and revisions in accordance with International Committee of Medical Journal Editors (ICMJE) criteria for authorship. The authors are guarantors of this document, which expresses the opinions and conclusions of the authors and not those of their corresponding affiliations. None of the authors received compensation from a pharmaceutical company, device company, or other agency for their contributions to the development of this manuscript.

\section{Availability of data and materials}

The datasets supporting the conclusions of this article are included within the article and have been validated by NAMSA; additional files can be provided upon request.

\section{Authors' contributions}

LG: drafting/revising the manuscript, study concept or design, analysis or interpretation of data, acquisition of data, and approval of the final draft. GE: drafting/revising the manuscript, acquisition of data, and approval of the final draft. AHC: drafting/revising the manuscript, analysis or interpretation of data, and approval of the final draft. CKM: analysis or interpretation of data, statistical analysis, and approval of the final draft. EL: drafting/revising the manuscript, study concept or design, analysis or interpretation of data, and approval of the final draft. PB: drafting/revising the manuscript, study concept or design, analysis or interpretation of data, acquisition of data and approval of the final draft.

\section{Competing interests}

LG has received consultancy and advisory fees from Allergan, Inc., and electroCore, LLC. GE has nothing to disclose. AHC has received advisory board fees from Depomed, Inc., Eli Lilly and Co., and Teva Pharmaceutical Industries Ltd. and speaker fees from Depomed, Inc., Merck \& Co., Inc., and Teva Pharmaceutical Industries Ltd.; Dr. Calhoun has also received research support from Autonomic Technologies, Inc., electroCore, LLC, and Scion NeuroStim, LLC. CKM is an employee of North American Science Associates Inc. EL is an employee of electroCore, LLC, and receives stock ownership. PB has received consultancy fees from Allergan, Inc., electroCore, LLC, Janssen Pharmaceuticals, Inc., and Lusofarmaco and advisory fees from Abbott Laboratories and Merck \& Co., Inc.

\section{Consent for publication}

Upon recruitment, all study subjects provided informed consent for publication of the dataset.

\section{Ethics approval and consent to participate}

Investigators obtained ethics approval for this study from the IRCCS, San Raffaele Pisana (identifier: SR_MM12/2014). Upon recruitment, all study subjects provided informed consent to participate.

\section{Author details \\ 'Headache Center, Carlo Besta Neurological Institute and Foundation, Via Celoria 11, 20133 Milan, Italy. ${ }^{2}$ Headache and Pain Unit, Istituto di Ricovero e Cura a Carattere Scientifico, San Raffaele Pisana, Via della Pisana 235, 00163 Rome, Italy. ${ }^{3}$ Carolina Headache Institute, 6114 Fayetteville Rd., Suite 109, 27713 Durham, NC, USA. ${ }^{4}$ North American Science Associates Inc., 4050 Olson Memorial Highway, Suite 450, 55422 Minneapolis, MN, USA. 5electroCore, LLC, 150 Allen Road, Suite 201, 07920 Basking Ridge, NJ, USA.}

Received: 24 August 2016 Accepted: 23 September 2016 Published online: 03 October 2016

\section{References}

1. Headache Classification Committee of the International Headache Society (2013) The International Classification of Headache Disorders, 3rd edition (beta version). Cephalalgia 33:629-808. doi:10.1177/0333102413485658

2. MacGregor EA (2008) Menstrual migraine. Curr Opin Neurol 21:309-315. doi:10.1097/WCO.0b013e3282fd185e

3. Vetvik KG, Macgregor EA, Lundqvist C, Russell MB (2014) Prevalence of menstrual migraine: a population-based study. Cephalalgia 34:280-288. doi:10.1177/0333102413507637
4. Mathew PG, Dun EC, Luo JJ (2013) A cyclic pain: the pathophysiology and treatment of menstrual migraine. Obstet Gynecol Surv 68:130-140. doi:10.1097/OGX.0b013e31827f2496

5. MacGregor EA (2014) A review of frovatriptan for the treatment of menstrual migraine. Int J Womens Health 6:523-535. doi:10.2147/JWWH.S63444

6. Nierenburg Hdel C, Ailani J, Malloy M, Siavoshi S, Hu NN, Yusuf N (2015) Systematic review of preventive and acute treatment of menstrual migraine. Headache 55:1052-1071. doi:10.1111/head.12640

7. Bigal M, Rapoport A, Aurora S, Sheftell F, Tepper S, Dahlof C (2007) Satisfaction with current migraine therapy: experience from 3 centers in US and Sweden. Headache 47:475-479. doi:10.1111/j.1526-4610.2007.00752.x

8. Silberstein SD (2000) Practice parameter: evidence-based guidelines for migraine headache (an evidence-based review): report of the Quality Standards Subcommittee of the American Academy of Neurology. Neurology 55:754-762

9. Pavlovic JM, Stewart WF, Bruce CA, Gorman JA, Sun H, Buse DC, Lipton RB (2015) Burden of migraine related to menses: results from the AMPP study. J Headache Pain 16:24. doi:10.1186/s10194-015-0503-y

10. Pinkerman B, Holroyd K (2010) Menstrual and nonmenstrual migraines differ in women with menstrually-related migraine. Cephalalgia 30:1187-1194. doi:10.1177/0333102409359315

11. Negro A, Napoletano F, Lionetto L, Marsibilio F, Sani G, Girardi P, Martelletti P (2014) Treatment of menstrual migraine: utility of control of related mood disturbances. Expert Rev Neurother 14:493-502. doi:10.1586/14737175.2014. 906304

12. Adelman JU, Calhoun A (2005) A randomized trial of frovatriptan for the intermittent prevention of menstrual migraine. Neurology 64:931, author reply

13. Calhoun A, Ford S (2008) Elimination of menstrual-related migraine beneficially impacts chronification and medication overuse. Headache 48: 1186-1193

14. Casolla B, Lionetto L, Candela S, D'Alonzo L, Negro A, Simmaco M, Martelletti P (2012) Treatment of perimenstrual migraine with triptans: an update. Curr Pain Headache Rep 16:445-451. doi:10.1007/s11916-012-0280-0

15. Sheftell FD, Feleppa M, Tepper SJ, Volcy M, Rapoport AM, Bigal ME (2004) Patterns of use of triptans and reasons for switching them in a tertiary care migraine population. Headache 44:661-668. doi:10.1111/j.1526-4610.2004.04124.x

16. Barbanti P, Grazzi L, Egeo G, Padovan AM, Liebler E, Bussone G (2015) Noninvasive vagus nerve stimulation for acute treatment of high-frequency and chronic migraine: an open-label study. J Headache Pain 16:61. doi:10.1186/ s10194-015-0542-4

17. Gaul C, Diener HC, Silver N, Magis D, Reuter U, Andersson A, Liebler EJ, Straube A, on behalf of the PREVA Study Group (2016) Non-invasive vagus nerve stimulation for PREVention and Acute treatment of chronic cluster headache (PREVA): A randomised controlled study. Cephalalgia 36:534-546. doi:10.1177/0333102415607070

18. Goadsby PJ, Grosberg BM, Mauskop A, Cady R, Simmons KA (2014) Effect of noninvasive vagus nerve stimulation on acute migraine: an open-label pilot study. Cephalalgia 34:986-993. doi:10.1177/0333102414524494

19. Kinfe TM, Pintea B, Muhammad S, Zaremba S, Roeske S, Simon BJ, Vatter H (2015) Cervical non-invasive vagus nerve stimulation (nVNS) for preventive and acute treatment of episodic and chronic migraine and migraineassociated sleep disturbance: a prospective observational cohort study. J Headache Pain 16:101. doi:10.1186/s10194-015-0582-9

20. Barbanti P, Aurilia C, Dall'Armi V, Egeo G, Fofi L, Bonassi S (2016) The phenotype of migraine with unilateral cranial autonomic symptoms documents increased peripheral and central trigeminal sensitization. A case series of 757 patients. Cephalalgia. doi:10.1177/0333102416630579.

21. Yang M, Rendas-Baum R, Varon SF, Kosinski M (2011) Validation of the Headache Impact Test (HIT-6) across episodic and chronic migraine. Cephalalgia 31:357-367. doi:10.1177/0333102410379890

22. Stewart WF, Lipton RB, Kolodner K (2003) Migraine disability assessment (MIDAS) score: relation to headache frequency, pain intensity, and headache symptoms. Headache 43:258-265

23. Lipton RB, Bigal ME, Ashina S, Burstein R, Silberstein S, Reed ML, Serrano D, Stewart WF, American Migraine Prevalence Prevention Advisory G (2008) Cutaneous allodynia in the migraine population. Ann Neurol 63:148-158. doi:10.1002/ana.21211

24. Dodick DW, Turkel CC, DeGryse RE, Diener HC, Lipton RB, Aurora SK, Nolan ME, Silberstein SD (2015) Assessing clinically meaningful treatment effects in controlled trials: chronic migraine as an example. J Pain 16:164-175. doi:10.1016/j.jpain.2014.11.004 
25. Silberstein SD, Marmura MJ, Shaw J, Yu S (2010) Headache prophylaxis with BoNTA: patient characteristics. Headache 50:63-70. doi:10.1111/j.1526-4610. 2009.01481.x

26. Meissner K, Fassler M, Rucker G, Kleijnen J, Hrobjartsson A, Schneider A, Antes G, Linde K (2013) Differential effectiveness of placebo treatments: a systematic review of migraine prophylaxis. JAMA Intern Med 173:1941-1951. doi:10.1001/jamainternmed.2013.10391

27. Eikermann-Haerter K, Kudo C, Moskowitz MA (2007) Cortical spreading depression and estrogen. Headache 47(Suppl 2):S79-S85. doi:10.1111/j.15264610.2007.00818.x

28. Sacco S, Ricci S, Degan D, Carolei A (2012) Migraine in women: the role of hormones and their impact on vascular diseases. J Headache Pain 13:177189. doi:10.1007/s10194-012-0424-y

29. Oshinsky ML, Murphy AL, Hekierski H Jr, Cooper M, Simon BJ (2014) Noninvasive vagus nerve stimulation as treatment for trigeminal allodynia. Pain 155:1037-1042. doi:10.1016/j.pain.2014.02.009

30. Hu Y, Guan X, Fan L, Jin L (2013) Triptans in prevention of menstrual migraine: a systematic review with meta-analysis. J Headache Pain 14:7. doi:10.1186/1129-2377-14-7

31. Merki-Feld GS, Imthurn B, Langner R, Sandor PS, Gantenbein AR (2013) Headache frequency and intensity in female migraineurs using desogestrelonly contraception: a retrospective pilot diary study. Cephalalgia 33:340346. doi:10.1177/0333102412473373

32. Coffee AL, Sulak PJ, Hill AJ, Hansen DJ, Kuehl TJ, Clark JW (2014) Extended cycle combined oral contraceptives and prophylactic frovatriptan during the hormone-free interval in women with menstrual-related migraines. J Womens Health (Larchmt) 23:310-317. doi:10.1089/jwh.2013.4485

33. MacGregor EA, Frith A, Ellis J, Aspinall L, Hackshaw A (2006) Prevention of menstrual attacks of migraine: a double-blind placebo-controlled crossover study. Neurology 67:2159-2163. doi:10.1212/01.wnl.0000249114.52802.55

34. Mannix LK, Savani N, Landy S, Valade D, Shackelford S, Ames MH, Jones MW (2007) Efficacy and tolerability of naratriptan for short-term prevention of menstrually related migraine: data from two randomized, double-blind, placebo-controlled studies. Headache 47:1037-1049. doi:10.1111/j.15264610.2007.00855.x

35. Calhoun AH, Pruitt AP (2015) Presenting a new, non-hormonally mediated menstrual headache: end-menstrual migraine [AHS abstract PS18]. Headache 55(suppl 3):127-187

36. Allais G, Sanchez del Rio M, Diener HC, Benedetto C, Pfeil J, Schauble B, van Oene J (2011) Perimenstrual migraines and their response to preventive therapy with topiramate. Cephalalgia 31:152-160. doi:10.1177/ 0333102410378049

37. Marmura MJ (2014) Safety of topiramate for treating migraines. Expert Opin Drug Saf 13:1241-1247. doi:10.1517/14740338.2014.934669

38. Rainero I, De Martino P, Rubino E, Vaula G, Gentile S, Pinessi L (2014) Noninvasive vagal nerve stimulation for the treatment of headache attacks in patients with chronic migraine and medication-overuse headache [AAN abstract]. Neurology 82(suppl 10):P1.262

\section{Submit your manuscript to a SpringerOpen ${ }^{\circ}$ journal and benefit from:}

- Convenient online submission

- Rigorous peer review

- Immediate publication on acceptance

- Open access: articles freely available online

- High visibility within the field

- Retaining the copyright to your article 